\title{
Irreversibilidad y pluralismo ontológico
}

\author{
Martín Labarca \& Olimpia Lombardi



RESUMEN

El problema de la irreversibilidad encuentra su origen a fines del siglo xIx y comienzos del siglo xx con los trabajos de Maxwell, Boltzmann y Gibbs. La controversia surge al intentar compatibilizar la evolución macroscópica irreversible de los sistemas termodinámicos con la dinámica t-invariante de sus constituyentes microscópicos. En la formulación tradicional de la mecánica estadística, la evolución irreversible se explica mediante la introducción de un grano grueso sobre la dinámica microscópica subyacente. Por este motivo, suele considerarse que la mecánica estadística sólo brinda una descripción subjetiva o meramente gnoseológica de la irreversibilidad. El propósito del presente trabajo consiste en impugnar las interpretaciones tradicionales de la irreversibilidad, poniendo de manifiesto que su único fundamento es la implícita adopción de un realismo metafísico que adjudica prioridad ontológica al mundo microscópico. Argumentaremos que las dificultades se disuelven cuando el problema es abordado sobre la base de un pluralismo ontológico de raíces kantianas, inspirado en el realismo internalista de Putnam. Desde esta perspectiva filosófica, la irreversibilidad macroscópica es una propiedad objetiva que no necesita ser explicada en términos microscópicos para adquirir legitimidad ontológica: el grano grueso que vincula ambas evoluciones no es más que la relación entre dos descripciones igualmente objetivas.

Palabras-GLAVe • Irreversibilidad. Mecánica estadística. Gibbs. Pluralismo ontológico. Grano grueso.

\section{INTRODUGGIÓN}

El origen del problema de la irreversibilidad se remonta al nacimiento de la mecánica estadística a fines del siglo xix y comienzos del siglo xx con los trabajos de Maxwell, Boltzmann y Gibbs. El propósito inicial de ese capítulo de la física fue el intento de explicar las propiedades y el comportamiento de los sistemas macroscópicos en función de las propiedades y el comportamiento de sus constituyentes microscópicos. Bajo el supuesto de que un sistema termodinámico se compone de partículas regidas por la mecánica clásica, la primera aplicación de la mecánica estadística consistió en el intento de dar cuenta de las propiedades y leyes termodinámicas en términos estrictamente mecánicos.

A pesar de haber transcurrido más de cien años desde sus primeras formulaciones, la mecánica estadística continúa presentando profundos escollos en su fundamentación. Entre ellos, el problema que ha generado las mayores dificultades es el que 
se refiere a la interpretación de la irreversibilidad termodinámica macroscópica. En efecto, el Segundo Principio de la Termodinámica expresa la evolución irreversible de todo sistema termodinámico cerrado hacia su estado final de equilibrio. Sin embargo, el comportamiento de los componentes mecánicos del sistema se encuentra regido por la mecánica clásica, cuya ley fundamental - la segunda ley de Newton - es tinvariante. El problema parece surgir, entonces, cuando se intenta compatibilizar la evolución macroscópica irreversible de los sistemas termodinámicos con la dinámica t-invariante de sus constituyentes microscópicos. En la formulación tradicional de la mecánica estadística, la evolución irreversible de los sistemas macroscópicos se explica mediante la introducción de un grano grueso en la dinámica microscópica subyacente. Por este motivo, suele considerarse que la mecánica estadística sólo brinda una descripción subjetiva o meramente gnoseológica de la irreversibilidad, que expresa nuestras limitadas capacidades de observación. Algunos autores, en un intento por preservar el carácter objetivo de la termodinámica, apelaron al concepto de emergencia que, si bien no hace de la irreversibilidad un fenómeno aparente o ilusorio, convierte a la ontología termodinámica en un dominio derivado y dependiente de la ontología mecánica fundamental.

El propósito del presente trabajo consiste en impugnar las interpretaciones tradicionales de la irreversibilidad, poniendo de manifiesto que su único fundamento es la implícita adopción de un realismo metafísico que adjudica prioridad ontológica al mundo microscópico. Argumentaremos que las dificultades en torno al problema de la irreversibilidad se disuelven cuando el problema es abordado sobre la base de un pluralismo ontológico de raíces kantianas, inspirado en el realismo internalista de Putnam. Desde esta perspectiva filosófica, la irreversibilidad macroscópica es una propiedad objetiva que no necesita ser explicada en términos microscópicos para adquirir legitimidad ontológica: el grano grueso que vincula las evoluciones macroscópica y microscópica no es más que la relación entre dos descripciones igualmente objetivas.

Con este propósito, comenzaremos por brindar una caracterización precisa del problema de la irreversibilidad a partir de la elucidación de los conceptos involucrados en su formulación. A continuación, describiremos el problema tal como se plantea en el caso particular de la mecánica estadística; sobre esta base podrán comprenderse las interpretaciones tradicionales de la irreversibilidad macroscópica, las cuales serán rechazadas desde una perspectiva tanto formal como histórica. Finalmente, argumentaremos a favor de un pluralismo ontológico de raigambre kantiana, subrayando el carácter no reductivo ni emergentista que adquieren las relaciones interteóricas desde esta perspectiva. Este marco filosófico nos permitirá admitir el carácter objetivo de la irreversibilidad y la autonomía ontológica del mundo termodinámico macroscópico respecto del dominio mecánico microscópico. 


\section{LA FORMULAGIÓN GENERAL DEL PROBLEMA DE LA IRREVERSIBILIDAD}

Cuando se discute el problema de la irreversibilidad, suelen confundirse o asimilarse dos nociones que, si bien emparentadas, son conceptualmente diferentes: $t$-invariancia e irreversibilidad (cf. Lombardi, 2006).

Definición 1: Una ley (ecuación) dinámica es t-invariante si es invariante ante la aplicación del operador de inversión temporal $\mathbf{T}$.

El operador T efectúa la transformación $t \rightarrow-t$ (donde $t$ es la variable que representa al tiempo) e invierte el signo de todas las variables dinámicas cuyas definiciones en función de $t$ son no invariantes ante la transformación $t \rightarrow-t .^{\mathbf{1}}$ Por ejemplo, en mecánica clásica de partículas la acción de $\mathbf{T}$ invierte el momento cinético pero no la posición de las partículas: $\mathbf{T} p=-p$ y $\mathbf{T} q=q$ (para el caso de otras teorías, cf. Earman, 2002). Como consecuencia, dada una ley t-invariante $L$, si $s(t)$ es una solución de $L$ (una evolución posible respecto de $L$ ), entonces $\mathbf{T} s(t)$ también lo es.

El concepto de irreversibilidad, por su parte, ha recibido diferentes caracterizaciones en la bibliografía sobre el tema. En general, se dice que una evolución es reversible cuando la secuencia de estados $s_{i}$ que la componen puede ocurrir en ambas direcciones temporales, $s_{\circ} \rightarrow s_{n}$ y $s_{n} \rightarrow s_{\circ}$; por lo tanto, en una evolución irreversible la secuencia de estados sólo puede ocurrir en un cierto orden temporal pero nunca en el orden inverso. ${ }^{2}$ En el caso de la irreversibilidad termodinámica, y en general en el ámbito de la física, la irreversibilidad se manifiesta a través de evoluciones que tienden a un estado final de equilibrio del cual el sistema no puede "escapar". Por lo tanto, el concepto de reversibilidad relevante puede definirse del siguiente modo:

Definición 2: Una evolución (solución de una ecuación dinámica) es reversible si no posee límite para $t \rightarrow \infty$.

Sobre la base de esas caracterizaciones, las evoluciones mecánicas en sistemas aislados (y finitos) son reversibles, puesto que son periódicas (o cuasi-periódicas). Las evoluciones termodinámicas en sistemas aislados, en cambio, son irreversibles, ya que

\footnotetext{
1 Por simplicidad, aquí no distinguiremos entre entidades matemáticas (ecuaciones y soluciones) y entidades físicas (leyes y evoluciones), utilizando ambas indistintamente.

2 Por supuesto, esta caracterización depende de cómo se interpreta el "puede ocurrir". Cuando la sucesión inversa no puede ocurrir porque nunca se dan ciertas condiciones iniciales, la irreversibilidad se considera una propiedad defacto. En el caso que nos ocupa, nos concentraremos en la irreversibilidad nomológica, en tanto propiedad de las evoluciones posibles respecto de una determinada ley dinámica.
} 
tienden hacia el equilibrio termodinámico, aumentando su entropía durante el proceso de acuerdo con el segundo principio.

No es necesario discutir los detalles de estas dos definiciones (cf. Albert, 2000; Arntzenius, 2004) para comprobar que los conceptos de t-invariancia y de reversibilidad son diferentes en la medida en que se aplican a entidades matemáticas (ecuaciones y soluciones) o físicas (leyes y evoluciones) diferentes. Mientras que la t-invariancia es una propiedad de ecuaciones dinámicas y, a fortiori, del conjunto de sus soluciones, la reversibilidad es una propiedad de una única solución. Ambas propiedades no se encuentran siquiera correlacionadas, pues pueden combinarse en los cuatro casos posibles: ecuaciones t-invariantes pueden tener soluciones irreversibles y ecuaciones no t-invariantes pueden tener soluciones reversibles (cf. Castagnino, Lara \& Lombardi, 2003; Castagnino \& Lombardi, 2005a, 2005b).

Una vez que se ha comprendido la diferencia entre los conceptos de t-invariancia y de reversibilidad, el problema de la irreversibilidad puede expresarse en términos de la siguiente pregunta: ¿cómo explicar evoluciones irreversibles mediante leyes t-invariantes? Con esta formulación del problema, en principio no existe obstáculo conceptual alguno para su solución: nada impide que una ecuación t-invariante tenga soluciones irreversibles. Sin embargo, las dificultades reaparecen debido a que las evoluciones mecánicas (tanto clásicas como cuánticas) no sólo son resultado de leyes t-invariantes, sino que son unitarias, esto es, pueden expresarse como:

$$
s(t)=U_{t} s(0)
$$

donde $U_{t}$ es un operador de evolución unitario de la forma $U_{t}=e^{-i G t}$, siendo $G$ el generador de la evolución que representa la energía del sistema. De ello se sigue que las evoluciones de la forma $s(t)=U_{t} s(\mathrm{O})$ son siempre reversibles, puesto que no tienen límite para $t \rightarrow \infty .^{3}$ En efecto, si $G$ representa la energía $\omega$ del sistema - que sólo adquiere valores reales -, la función $e^{-i \omega t} s(\mathrm{o})$ no tiene límite para $t \rightarrow \infty$, puesto que la exponencial compleja $e^{-i \omega t}$ es una función "oscilante". Esto significa que, si la dinámica microscópica de un sistema se expresa a través de evoluciones unitarias, tales evoluciones nunca tienden a un estado final de equilibrio. Por lo tanto, cuando el estado macroscópico del sistema se define en términos del estado microscópico, el desafío consiste en dar cuenta de la evolución irreversible y no unitaria de tal macroestado hacia el equilibrio. 
En definitiva, a diferencia de lo que suele suponerse, el verdadero escollo para explicar la irreversibilidad macroscópica no reside en la t-invariancia de las leyes microscópicas, sino en el carácter unitario de las evoluciones mecánicas del micronivel. El problema de la irreversibilidad consiste, entonces, en explicar evoluciones macroscópicas irreversibles y no unitarias en términos de evoluciones microscópicas reversibles y unitarias.

\section{EL PROBLEMA DE LA IRREVERSIBILIDAD EN MECÁNICA ESTADÍSTICA}

Históricamente, el problema de la irreversibilidad surge a fines del siglo xIX con la adopción de la teoría cinética de los gases: si un gas no es más que un conjunto de partículas en interacción, sus propiedades termodinámicas macroscópicas deberían poder explicarse como resultado de las propiedades mecánicas de sus partículas componentes. En particular, se impone el problema de explicar la irreversibilidad termodinámica macroscópica en términos de las evoluciones mecánicas del nivel subyacente. A la luz de este problema, Boltzmann y Gibbs, entre otros, formulan la mecánica estadística clásica como una nueva teoría que, al combinar las leyes de la mecánica clásica con procedimientos estadísticos, resulta particularmente adecuada para el estudio de sistemas compuestos por un número tan alto de partículas que resulta estéril todo intento de cálculo preciso mediante la mecánica clásica. En la actualidad, la mecánica estadística de Gibbs constituye una herramienta teórica estándar de la física por sus múltiples aplicaciones y éxitos predictivos. ${ }^{4}$ Sin embargo, aún en nuestros días, su validez sigue siendo severamente cuestionada por algunos autores debido al tipo de explicación de la irreversibilidad macroscópica que suele inferirse de su formulación teórica (cf. Lombardi, 2003a).

Para dar cuenta del comportamiento dinámico de un sistema compuesto por un elevado número de partículas, la estrategia de Gibbs se basa en describir el comportamiento del ensemble representativo del sistema bajo estudio, esto es, un conjunto de sistemas abstractos, conceptualmente construidos, que poseen la misma microestructura que el sistema de interés y están sometidos a los mismos vínculos externos, pero se encuentran distribuidos sobre los diferentes microestados compatibles con los valores de las variables macroscópicas del sistema. El microestado instantáneo de cada sistema del ensemble se representa mediante un punto en el espacio de las fases $\Gamma$; la situación

4 Uno de los problemas de fundamentación de la mecánica estadística reside en la existencia de dos enfoques teóricos, el de Boltzmann y el de Gibbs, difícilmente reconciliables. Aquí nos concentraremos sólo en el enfoque de Gibbs por tratarse de una herramienta teórica estándar en la práctica científica (cf. Lombardi \& Labarca, 2005a). 
del ensemble como un todo queda representada por una "nube" de tales puntos representativos, uno por cada sistema del ensemble 5 (ver figura 1).

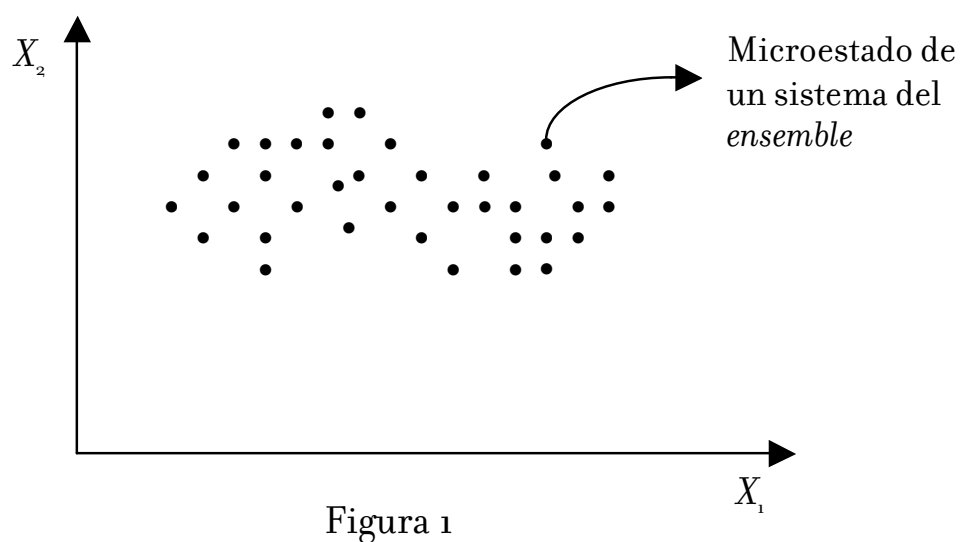

Si el número de sistemas del ensemble es suficientemente alto, la situación del ensemble en cada instante puede especificarse mediante la densidad $\rho(x, t)$ de distribución de los puntos representativos en el espacio de las fases. En general, la función $\rho$ se considera normalizada a la unidad; en este caso, $\rho$ es una medida que brinda la probabilidad por unidad de volumen de que un punto representativo de un sistema cualquiera del ensemble se encuentre en las diferentes regiones del espacio de las fases (ver figura 2).

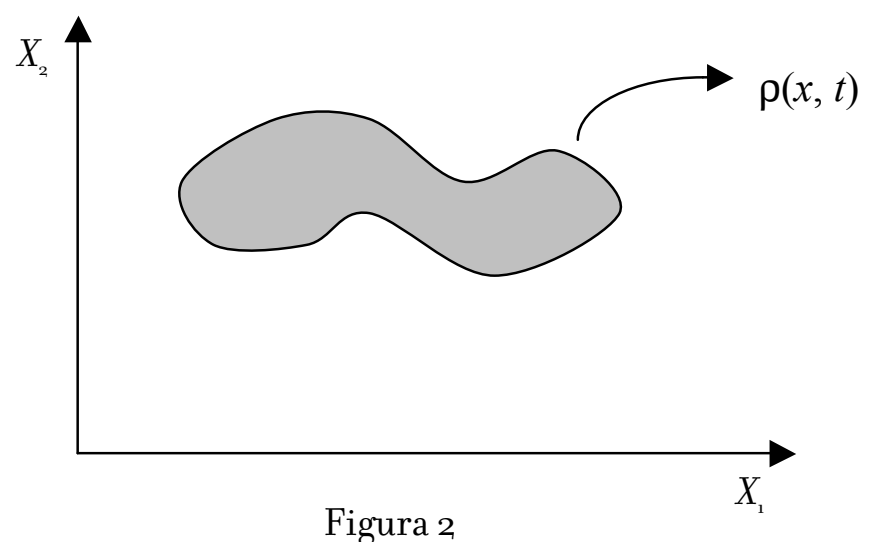

5 El espacio de las fases correspondiente a un sistema es un espacio abstracto de tantas dimensiones como variables de estado posea el sistema. 
Gibbs define equilibrio estadístico como la situación en la cual la probabilidad representada por la función $\rho$ es independiente del tiempo. Un modo sencillo de asegurar el equilibrio estadístico en un sistema aislado es construir un ensemble para el cual $\rho$ se distribuye uniformemente sobre toda la región accesible $\Gamma_{\mathrm{A}}$ del espacio de las fases $\Gamma$, donde $\Gamma_{\mathrm{A}}$ está determinada por la energía del sistema (que es constante con el tiempo puesto que se trata de un sistema aislado) y los vínculos $C$ impuestos exteriormente (por ejemplo, el volumen del recipiente que contiene al sistema). Gibbs denomina "ensemble microcanónico" a este tipo de ensemble, definido como:

$$
\begin{cases}\rho(x)=c t e & \forall x \in \Gamma_{\mathrm{A}} \\ \rho(x)=0 & \forall x \notin \Gamma_{\mathrm{A}}\end{cases}
$$

Esto significa que, en la situación microcanónica, los sistemas que componen el ensemble pueden encontrarse con igual probabilidad en cualquiera de los microestados representados por los puntos incluidos en $\Gamma_{\mathrm{A}}$. A su vez, en esta situación, la entropía de Gibbs $S_{G}$ se define en términos de la función $\rho(x)$ (cf. Lombardi, 2003b; Lombardi \& Labarca, 2005a):

$$
S_{\mathrm{G}}=-k \int_{\Gamma} \rho(x) \log \rho(x) \mathrm{d} \Gamma
$$

A fin de conectar esta descripción mecánico-estadística con la descripción termodinámica, el enfoque de Gibbs establece los nexos entre ciertas propiedades del ensemble representativo y ciertas propiedades termodinámicas del sistema bajo estudio. En particular, el equilibrio estadístico se vincula con el equilibrio termodinámico y, por lo tanto, la entropía de $\mathrm{Gibbs}_{\mathrm{G}}$ pasa a concebirse como una expresión de la entropía termodinámica en términos microscópicos. Veamos cómo se establece tal vínculo.

Supóngase un gas confinado en una pequeña zona de un recipiente aislado mediante un tabique divisor. Si en $t=0$ se retira el tabique, veremos que el gas se difunde hasta ocupar todo el volumen del recipiente. La termodinámica da cuenta de este fenómeno diciéndonos que el sistema, inicialmente en equilibrio termodinámico, pasa a estar fuera del equilibrio al retirarse el tabique, y evoluciona irreversiblemente hacia un nuevo estado de equilibrio termodinámico aumentando su entropía termodinámica durante el proceso (ver figura 3 ). 


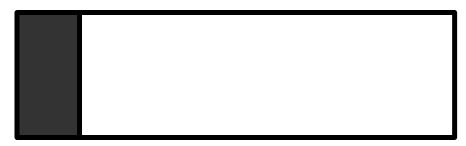

$t<0$

equilibrio

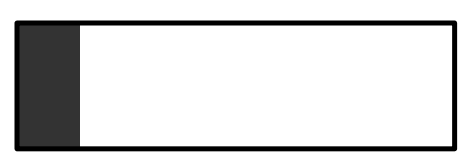

$t=0$

no equilibrio


nuevo

equilibrio

Figura 3

¿Cómo dar cuenta de este proceso en términos mecánico-estadísticos mediante el enfoque de Gibbs? Antes de retirar el tabique, el sistema está sometido a ciertos vínculos $C_{\circ} \mathrm{y}$, dado que se encuentra en equilibrio estadístico, queda representado por un ensemble microcanónico $\rho(x)$ definido por:

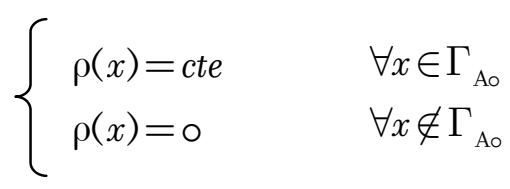

donde $\Gamma_{\text {Ao }}$ es la región accesible del espacio de las fases determinada por los vínculos $C_{\circ}$ (entre ellos, el volumen de la pequeña zona del recipiente donde inicialmente se encuentra confinado el gas). Cuando en $t=0$ se retira el tabique divisor, el ensemble microcanónico $\rho(x)$ se convierte en un ensemble de no equilibrio representado por $\rho_{\circ}(x, t=0)$, que adopta valores no nulos en $\Gamma_{\text {Ao }}$, pero para el cual la nueva región accesible del espacio de las fases, determinada por los nuevos vínculos $C_{1}$ (en particular, el nuevo volumen que ocupa el gas al haberse retirado el tabique), es $\Gamma_{\mathrm{Al}_{\mathrm{l}}}>\Gamma_{\mathrm{Ao}}$. A su vez, en la situación final el sistema debería adquirir un nuevo estado de equilibrio, ahora representado por un ensemble microcanónico $\rho_{1}(x)$ definido por:

$$
\begin{cases}\rho_{1}(x)=\text { cte } & \forall x \in \Gamma_{\mathrm{Al}_{1}} \\ \rho_{1}(x)=0 & \forall x \notin \Gamma_{\mathrm{Al}}\end{cases}
$$


Por lo tanto, entre el instante $t=0$ en que se retira el tabique y el instante final en que se alcanza el nuevo estado de equilibrio debería darse la evolución

$$
\rho_{\mathrm{o}}(x, t=0) \rightarrow \rho_{\mathrm{o}}(x, t)=\rho_{1}(x):
$$

el ensemble de no equilibrio inicial $\rho_{\circ}(x, t=0)$, que adopta valores no nulos sólo en $\Gamma_{\text {Ao }}$, debería convertirse en el ensemble microcanónico final $\rho_{1}(x)$, uniforme sobre la nueva región accesible $\Gamma_{\mathrm{Al}}$ (ver figura 4).



Figura 4

El núcleo del problema clásico de la irreversibilidad reside en que tal evolución es físicamente imposible de acuerdo con las leyes mecánicas que rigen el comportamiento de cada sistema del ensemble. En efecto, un resultado central de la mecánica clásica es el teorema de Liouville, según el cual el volumen de la región en la que la función densi$\operatorname{dad} \rho(x)$ adopta valores no nulos no varía a través de la evolución (ver figura 5 ).

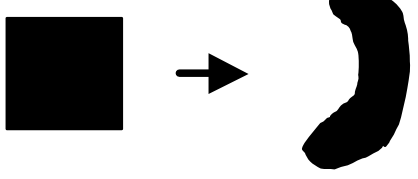

Figura 5

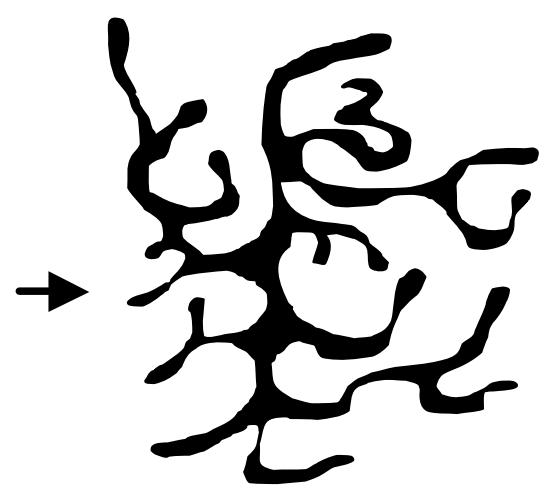

$$
5
$$


Esto significa que la región inicial podrá deformarse y extenderse en la nueva región accesible del espacio de las fases $\Gamma_{\mathrm{Al}_{1}}$, pero, al mantener su volumen constante, no podrá cubrir de un modo efectivo tal región correspondiente al nuevo estado de equilibrio. A su vez, dado que el volumen de la región en la que la función densidad $\rho(x)$ adopta valores no nulos es constante en el tiempo, la entropía de Gibbs, definida mediante una integral de tal función sobre el espacio de las fases, también se mantiene constante a través de toda la evolución y, en consecuencia, no puede considerarse una adecuada expresión de la entropía termodinámica.

Este problema puede ser visto desde una perspectiva formal recordando el carácter unitario de los procesos mecánicos. En efecto, puesto que cada uno de los sistemas del ensemble evoluciona según las leyes de la mecánica clásica, la función densidad $\rho(x, t)$ - que representa el comportamiento temporal del ensemble como un todo-también evoluciona unitariamente como:

$$
\rho(x, t)=U_{t} \rho(x, t=0)
$$

donde $U_{t}$ es un operador de evolución unitario. Por lo tanto, $\rho(x, t)$ no tiene límite para $t \rightarrow \infty$ y, en consecuencia, nunca alcanza un estado final de equilibrio a través de un proceso irreversible.

¿Cómo explicar, entonces, la irreversibilidad macroscópica? Según la explicación gibbsiana, lo que en realidad sucede es que, dada la alta inestabilidad del sistema, la región inicial ocupada por $\rho_{\circ}(x, t=0)$ se ha distribuido y ramificado hasta el punto de cubrir de un modo aparentemente uniforme la nueva región $\Gamma_{\mathrm{Al}}$ correspondiente al nuevo estado de equilibrio (ver figura 6).

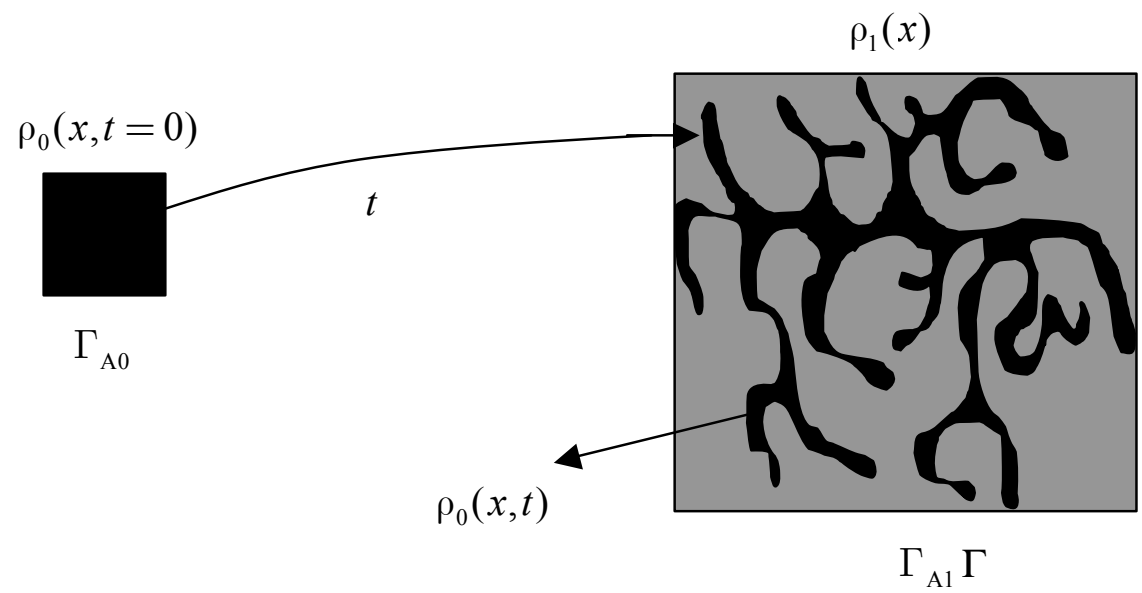

Figura 6 
A fin de dar cuenta de la creciente deformación inicial, puede definirse una distribución de grano grueso $\rho_{c g}(x)$ : si se divide el espacio de las fases $\Gamma$ en celdas $C_{i}$ de igual volumen, para cada celda $C_{i}$, la distribución $\rho_{c g}(x)$ tendrá valor nulo si $\rho_{\circ}(x)=0$ para todo $x$ perteneciente a $C_{i}$, y adoptará un valor no nulo en $C_{i}$ si $\rho_{\mathrm{o}}(x) \neq 0$, para algún $x$ perteneciente a $C_{i}$ (ver figura 7$)$.

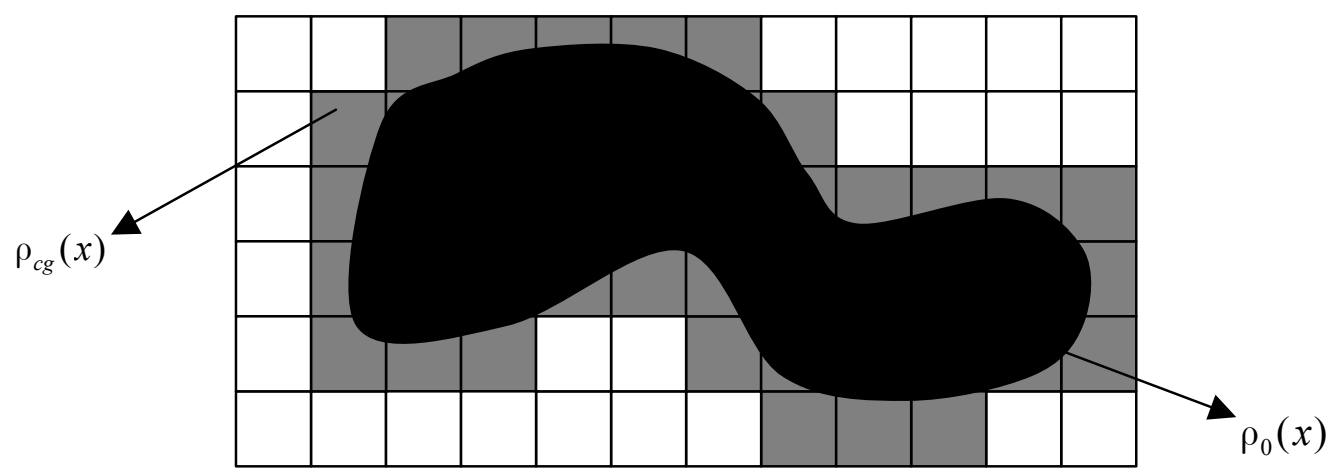

Figura 7

Queda claro que, si bien el volumen de la región en la que $\rho_{\mathrm{o}}(x, t)$ adopta valores no nulos no varía con el tiempo, puede esperarse que el volumen de la región en la que $\rho_{c g}(x, t)$ adopta valores no nulos aumente a través de la evolución a medida que la región inicial, al deformarse, vaya ingresando en mayor cantidad de celdas. Si esto ocurre, $\rho_{c g}(x, t)$ tenderá, para $t \rightarrow \infty$, a un valor final de equilibrio $\rho_{c g(e q)}$ constante sobre toda la nueva región accesible del espacio de las fases:

$$
\begin{cases}\rho_{c g(e q)}=c t e & \forall x \in \Gamma_{\mathrm{Al}} \\ \rho_{c g(e q)}=0 & \forall x \notin \Gamma_{\mathrm{Al}}\end{cases}
$$

Formalmente esto significa que, aunque $\rho_{\mathrm{o}}(x, t)$ evoluciona unitariamente y, por tanto, no tiene límite para $t \rightarrow \infty, \rho_{c g}(x, t)$ sí puede tener un límite definido. Puede demostrarse que, bajo condiciones de alta inestabilidad muy precisas, ${ }^{6}$ la evolución de $\rho_{c g}(x, t)$ es irreversible y tiende a un valor final de equilibrio:

$$
\lim _{t \rightarrow \infty} \rho_{c g}(x, t)=\rho_{c g(e q)}
$$

6 En particular, esto se cumple cuando el sistema es mezclador. La propiedad de mezcla está definida con precisión en el marco de la teoría ergódica. 
A su vez, mediante $\rho_{c g}(x, t)$ puede definirse una entropía de grano grueso $S_{c g}$ tal que:

$$
S_{c g}(t)=-k \int_{\Gamma} \rho_{c g}(x, t) \log \rho_{c g}(x, t) \mathrm{d} \Gamma
$$

Si la distribución de grano grueso $\rho_{c g}(x, t)$ tiene límite para $t \rightarrow \infty$, lo mismo ocurrirá $\operatorname{con} S_{c g}(t)$ :

$$
\lim _{t \rightarrow \infty} S_{c g}(t)=S_{c g(e q)}
$$

En otras palabras, la entropía de grano grueso manifiesta una evolución irreversible hacia su valor máximo de equilibrio, propia de la entropía termodinámica.

\section{LAS INTERPRETAGIONES TRADIGIONALES DE LA IRREVERSIBILIDAD}

Como hemos visto, en la mecánica estadística de Gibbs, la evolución irreversible de un sistema hacia el equilibrio termodinámico se explica mediante la introducción de un grano grueso sobre la dinámica microscópica. Por este motivo, suele afirmarse que este enfoque teórico sólo brinda una descripción subjetiva de la irreversibilidad. Desde esa perspectiva se argumenta que, si un observador "perfecto" describiera la evolución del sistema inicialmente fuera del equilibrio a través del comportamiento de suensemble representativo, observaría la creciente distorsión y ramificación en el espacio de las fases de la región inicial, pero podría comprobar la validez del teorema de Liouville: nunca se alcanza una distribución uniforme sobre la región asociada al nuevo estado de equilibrio, pues el volumen de la región inicial permanece invariante durante toda la evolución. Por lo tanto, la evolución irreversible hacia el equilibrio no sería más que una apariencia debida exclusivamente a nuestros limitados poderes de observación.

La interpretación subjetivista de la irreversibilidad ${ }^{7}$ se basa implícitamente en un supuesto ontológico reduccionista, según el cual un sistema termodinámico macroscópico "no es más" que un conjunto de partículas regidas por la mecánica clásica. Tal supuesto subyace a la lectura que la propia física suele brindar de sus propios resultados teóricos; como afirma Rohrlich:

\footnotetext{
7 En la bibliografía sobre el tema suele hablarse de una interpretación subjetivista o subjetiva de la irreversibilidad. Sin embargo, sería más correcto hablar de una interpretación gnoseológica pues, desde esa perspectiva, la irreversibilidad no depende de un sujeto particular, sino de las capacidades de acceso a la realidad de cualquier observador humano.
} 
la química nos dice que un pedazo de madera es 'realmente' un complicado arreglo de muchos tipos de moléculas unidas entre sí; la física atómica nos dice que las moléculas son "realmente" varios átomos mantenidos juntos por fuerzas atómicas; la teoría de partículas nos dice que los átomos son "realmente" partículas elementales en interacción, y así sucesivamente (1988, p. 295-6).

En el caso de la irreversibilidad macroscópica, esta interpretación subjetivista ha adquirido una aceptación sorprendentemente amplia. Por ejemplo, ya Heisenberg escribía:

Gibbs fue el primero en introducir un concepto físico que sólo puede aplicarse a un objeto cuando nuestro conocimiento del objeto es incompleto. Si, por ejemplo, el movimiento y la posición de cada molécula de un gas fueran conocidos, entonces no tendría sentido continuar hablando de la temperatura del gas (1958, p. 38).

De un modo similar, Born afirmaba: "La irreversibilidad es, por lo tanto, una consecuencia de la explícita introducción de la ignorancia en las leyes fundamentales" (1949, p. 72). Esta idea ha perdurado hasta nuestros días; por ejemplo, en un reciente libro de texto aún se lee: "La irreversibilidad aparece sólo porque el estado instantáneo del sistema no puede conocerse con infinita precisión" (Vauclair, 1993, p. 198).

Es interesante señalar que incluso quienes se resisten a admitir el carácter subjetivo de la irreversibilidad macroscópica adoptan una interpretación subjetivista de la explicación gibbsiana; precisamente por este motivo se oponen al enfoque de Gibbs. En este sentido, ya Popper mostraba su rechazo:

Es claramente absurdo creer que los peniques caen o las moléculas entran en colisión de manera aleatoria porque nosotros no conocemos las condiciones iniciales, y que actuarían de modo diferente si algún demonio nos revelase su secreto: no es sólo imposible, es absurdo explicar las frecuencias estadísticas objetivas por medio de la ignorancia subjetiva (1982 [1956], p. 106).

Por ello, Popper proponía una explicación objetiva de los procesos irreversibles basada en su interpretación propensivista de la probabilidad. En nuestros días, Bricmont (1995) también dirige sus críticas al enfoque gibbsiano, sosteniendo que todo intento de "forzar" el aumento de la entropía de Gibbs mediante particiones de grano grueso genera la incorrecta impresión de que la irreversibilidad depende de tales particiones y, por lo tanto, es un fenómeno subjetivo. Pero tal vez el más ferviente opositor a la 
interpretación subjetivista de la irreversibilidad sea Prigogine, quien considera que "en la imagen clásica, la irreversibilidad era debida a nuestras aproximaciones, a nuestra ignorancia" (1994, p. 37). Por ello, concentra sus ataques en la explicación gibbsiana de la irreversibilidad:

esta interpretación se convierte en absurda tan pronto como se dejan de lado las asociaciones irrelevantes con problemas tecnológicos $[\ldots]_{i}$ La afinidad química, la conducción calorífica, la viscosidad y todas las propiedades asociadas a la producción irreversible de entropía dependerían, de esta manera, del observador! La combustión que tiene lugar en el horno, ¿no se debería al aumento de nuestra ignorancia? (Prigogine \& Stengers, 1983 [1979], p. 24, ${ }^{\mathbf{8}}$

Sin duda, estos argumentos dirigidos contra la interpretación subjetivista de la irreversibilidad macroscópica suenan muy convincentes. No parece razonable pensar que los fenómenos irreversibles que observamos, como la expansión de un gas inicialmente confinado en la mitad de un recipiente cerrado, o la uniformización de la temperatura en un cuerpo aislado con un gradiente térmico inicial, dependan de lo que el observador conozca o no sobre el sistema. Incluso si el demonio del que nos habla Popper nos susurrara al oído la posición y la velocidad exactas de cada una de las moléculas confinadas en la pequeña región inicial del recipiente, ello no afectaría en absoluto la evolución futura del gas. Frente a estas dificultades, se ha intentado recuperar el carácter objetivo de la descripción termodinámica postulando la emergencia de la irreversibilidad macroscópica y, en general, de losítems termodinámicos como temperatura, presión o entropía. El concepto de emergencia se presenta como una noción que, evitando el eliminativismo reductivo, conseguiría explicar cómo ciertos ítems ontológicos "surgen" a partir de otros más básicos (cf., por ejemplo, Bunge, 1982; Hendry, 1999; Luisi, 2002): los ítems emergentes, si bien objetivamente existentes y no meramente aparentes o ilusorios, deben su objetividad a los ítems más básicos de los cuales emergen.

El concepto de emergencia ha sido discutido ampliamente en la bibliografía de las últimas décadas, dando lugar a múltiples caracterizaciones a menudo divergentes. En su acepción sincrónica, ${ }^{9}$ la emergencia se refiere a la relación que existe, en cada

8 Para un análisis de los distintos aspectos de esta posición, cf. Lombardi, 1998, 1999, 2000.

9 Como afirma El Hani (2004), es necesario distinguir entre emergencia sincrónica y emergencia diacrónica. La emergencia diacrónica se refiere al surgimiento de nuevas entidades y/o propiedades en la evolución temporal de un sistema. Puesto que aquí estamos interesados en el problema sincrónico de la relación entre las ontologías descritas por teorías aceptadas simultáneamente por la comunidad científica, centraremos nuestra atención exclusivamente en la noción de emergencia sincrónica. 
instante, entre las entidades, las propiedades y el comportamiento de un sistema y las características propias de su microestructura. En este sentido, el término "emergencia" y sus derivados han sido aplicados a ítems pertenecientes a muy diferentes categorías ontológicas: objetos individuales, propiedades, relaciones, hechos, estructuras, eventos, procesos, comportamientos etc. En el ámbito de la física, entre los ejemplos típicos de ítems considerados emergentes se encuentran la conductividad eléctrica, la viscosidad, la estructura moleculary, paradigmáticamente, los referentes de la termodinámica. Sin embargo, a pesar del relativo acuerdo acerca de los ítems que pueden ser calificados como emergentes, las teorías de la emergencia se presentan bajo muy diferentes formas, difícilmente subsumibles bajo una visión unificada. No obstante, es posible reconocer al menos dos notas comunes a las diferentes propuestas emergentistas.

En primer lugar, salvo en el caso de concepciones sumamente débiles del concepto de emergencia, el emergentismo se presenta como una posición no reduccionista: la caracterización de los ítems emergentes no puede ser deducida, explicada o predicha a partir de la descripción completa de ciertos otros ítems considerados más básicos. A pesar de que esta nota suele ser formulada en términos epistémicos, en general se la concibe como expresando un hecho ontológico y no sólo las limitaciones epistémicas del ser humano. En este sentido, se trata de una imposibilidad - de deducir, explicar o predecir - en principio; a ello ya se refería Broad cuando, con resonancias laplaceanas, afirmaba que ni siquiera un arcángel matemático podría deducir, explicar o predecir lo emergente a partir de las características de los ítems más básicos (cf. Broad, 1925, p. 70). Sobre esta base, el emergentismo defiende el carácter real y objetivo de los ítems emergentes, a diferencia del reduccionismo ontológico tradicional.

La segunda nota común a las diferentes posiciones emergentistas consiste en concebir la relación de emergencia como una relación asimétrica. En efecto, si un ítem A emerge a partir de un ítem (o un conjunto de ítems) B, entonces B no emerge de A. En algunos casos, la emergencia es caracterizada en términos de implicación: la descripción completa de B ofrece condiciones necesarias pero no suficientes para la derivación de la descripción de A (cf. Atmanspacher \& Bishop, 2007). Ahora bien, si la emergencia se concibe como una relación en el plano ontológico, su asimetría también debe expresar una cierta diferencia ontológica básica entre los ítems involucrados. En efecto, el carácter asimétrico de la relación de emergencia supone que los ítems emergentes poseen una existencia secundaria o derivada, dependen ontológicamente de los ítems respecto de los cuales emergen. No se afirma, entonces, que los ítems emergentes no existan en un sentido absoluto, sino que dependen del dominio cuya prioridad ontológica hace posible su existencia. En otras palabras, la realidad se organiza jerárquicamente a partir de un estrato ontológico fundamental, del cual surgen las ontologías emergentes. Desde esta perspectiva, la irreversibilidad macroscópica se 
interpreta como una propiedad emergente que depende del comportamiento del estrato fundamental, constituido por las partículas microscópicas regidas por la mecánica clásica.

\section{Dos ARGUMENTOS GONTRA LAS INTERPRETAGIONES TRADIGIONALES}

Si bien claramente diferentes, la interpretación subjetivista y la interpretación emergentista de la irreversibilidad coinciden en adjudicar prioridad ontológica al dominio microscópico, sea negando la existencia objetiva de la irreversibilidad termodinámica, sea postulando su carácter emergente. Este supuesto metafísico tiene, sin duda, numerosos antecedentes en la historia de la filosofía. No obstante, es también propio de la filosofía cuestionar incluso los supuestos más arraigados. En este caso, entonces, cabe preguntarse por los motivos que justifican postular la existencia de un dominio fundamental de la realidad del cual depende ontológicamente toda otra existencia: ¿puede apelarse a razones formales, derivadas de la estructura de las teorías científicas involucradas? ¿Hay motivaciones fundadas en el propio desarrollo histórico de la ciencia?

Como hemos visto, en el caso del problema de la irreversibilidad nos encontramos ante dos descripciones, la de grano fino $\rho(t)$ y la de grano grueso $\rho_{c g}(t)$, que adjudican al sistema propiedades contradictorias, esto es, reversibilidad e irreversibilidad respectivamente. También hemos señalado que, frente a esta situación, la posición tradicional consiste en considerar que el grano grueso representa la discriminación necesariamente finita con la que determinamos empíricamente el valor del estado $\rho(t)$ mediante nuestros instrumentos de medición: la evolución de grano grueso sería, entonces, una representación que expresaría únicamente nuestra "ignorancia" subjetiva, resultado de nuestros limitados poderes de observación.

El carácter aparentemente inevitable de esta conclusión se diluye cuando la operación matemática de grano grueso se concibe en términos de proyección. Una proyección es la operación que efectúa un operador proyección o proyector, esto es, un operador $\Pi$ para el que se cumple $\Pi^{2}=\Pi$. Esto significa que, una vez realizada la primera proyección, toda aplicación subsiguiente del proyector no modifica el resultado original: $\Pi \Pi a=\Pi a$ (piénsese en la proyección de un vector sobre un eje coordenado: una nueva proyección conduce al mismo vector obtenido inicialmente). Ahora bien, toda operación de grano grueso define un proyector (cf. Mackey, 1989), de modo tal que la descripción de grano grueso $\rho_{c g}$ puede definirse como una proyección de la descripción de grano fino $\rho$ :

$$
\rho_{c g}=\Pi \rho
$$


En nuestro ejemplo, para cada celda $C_{i}$, el proyector adjudica a $\rho_{c g}$ el valor o cuando $\rho$ es nulo en todos los puntos pertenecientes a $C_{i}$, y un valor no nulo cuando $\rho$ es no nulo en algún punto de $C_{i}$.

En principio, el reconocimiento de la equivalencia entre las operaciones de grano grueso y de proyección no parece atentar contra la tradicional interpretación subjetivista o gnoseológica del grano grueso. En efecto, cuando un vector $\vec{a}$ perteneciente a un cierto espacio (por ejemplo, de dos dimensiones) se proyecta sobre un subespacio de dicho espacio (por ejemplo, de una dimensión) obteniéndose un vector $\vec{b}$, parece producirse una suerte de "pérdida de información" al pasar de $\vec{a}$ a $\vec{b}$ (ver figura 8).

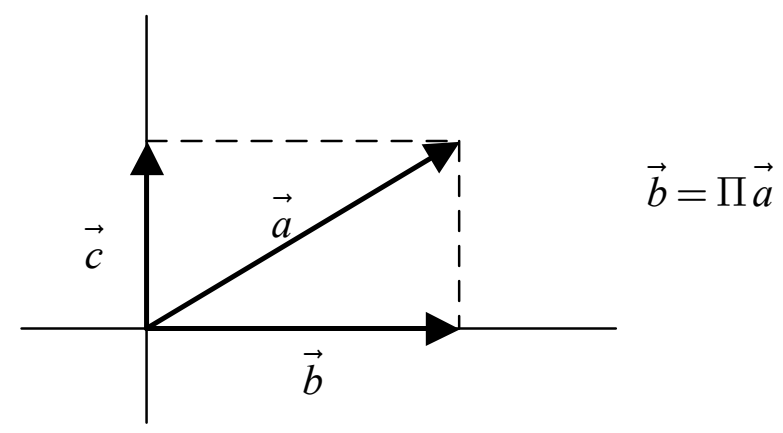

Figura 8

En otras palabras, parece natural pensar que el vector $\vec{a}$ representa la magnitud fundamental, puesto que el vector $\vec{b}$ es sólo una componente (una "sombra") de $\vec{a}$ : necesitamos otro vector $\vec{c}$ para reconstruir $\vec{a}$. Sin embargo, esta conclusión no toma en cuenta que, para hacer esta proyección, hemos decidido previamente "embeber" el vector $\vec{a}$ en un cierto espacio y el vector $\vec{b}$ en un subespacio de aquél; pero también podríamos haber decidido efectuar la proyección inversa, según la cual $\vec{b}$ es el vector proyectado y $\vec{a}$ es el resultado de la proyección (ver figura 9).



Figura 9 
Esto significa que, dados dos vectores cualesquiera $\vec{a}$ y $\vec{b}$, podemos embeber $\vec{a}$ en un espacio de $N$ dimensiones y $\vec{b}$ en un espacio de $M<N$ dimensiones, y considerar que $\vec{b}$ es la proyección de $\vec{a}$, o viceversa: al decidir en qué espacios embeber los vectores, hemos elegido la dirección de la proyección. Pero el aspecto central de esta cuestión es que ambas operaciones son igualmente legítimas desde un punto de vista matemático. En otras palabras, nada hay intrínsecamente "más fundamental" en un vector respecto del otro, no hay "pérdida de información" al pasar de uno a otro. Cuando elegimos la dirección de la proyección, estamos implícitamente presuponiendo el carácter fundamental de uno de los vectores; pero de igual modo podríamos haber escogido la dirección inversa, y el carácter fundamental se habría intercambiado de un vector al otro.

La prioridad que suele asignarse a ciertas descripciones, consideradas reflejo de la "verdadera" ontología, lejos de ser "natural" o impuesta por motivos formales, en general resulta de contingencias históricas o de convicciones metafísicas explícitas o implícitas. Por ejemplo, el enorme éxito de la mecánica clásica, formalizada mediante los métodos continuos del cálculo infinitesimal, influyó decisivamente en que se impusiera la idea de que las magnitudes físicas son "realmente" continuas y de que natura non facit saltus. Cuando, en nuestros días, los físicos apelan a simulaciones numéricas en sus computadoras, trabajan sistemáticamente con funciones discretas; no obstante, siguen convencidos de que tales técnicas son meras aproximaciones a la "verdadera" realidad continua. Sería interesante imaginar una historia contrafáctica donde las computadoras se hubieran inventado antes que el cálculo infinitesimal: tal vez, en ese caso, las técnicas continuas se habrían concebido como meras aproximaciones de la "verdadera" realidad discreta.

En otros casos, ciertas teorías son consideradas fundamentales debido a que describen una ontología microscópica: la mecánica clásica es más fundamental que la termodinámica, la mecánica cuántica es más fundamental que la mecánica clásica, y así sucesivamente. Este supuesto se basa en un presupuesto metafísico reduccionista que impone una dirección privilegiada a la proyección. Sin embargo, algunas posiciones "no ortodoxas" han intentado desafiar la prioridad ontológica tradicionalmente adjudicada al dominio microscópico. Por ejemplo, en sus trabajos de las décadas de los 1970 y de los 1980, Ilya Prigogine y otros miembros de su grupo de investigación defendieron el carácter fundamental de las teorías macroscópicas supuestamente fenomenológicas, como la termodinámica, sobre la base de la convicción de que el indeterminismo y la irreversibilidad presentes en el macronivel no pueden ser meras apariencias subjetivas; a la luz de esta idea, dirigieron sus esfuerzos teóricos a rechazar todo vínculo de grano grueso, y a demostrar que la descripción microscópica puede obtenerse como un límite ideal de la descripción macroscópica fundamental (cf. Misra, Prigogine \& Courbage, 1979; Courbage \& Prigogine, 1983). 
Si no hay razones formales que respalden el supuesto de la prioridad ontológica de un dominio - el dominio microscópico en el caso de la irreversibilidad - respecto del resto de los dominios de lo real, tal vez puedan hallarse motivos filosóficos respaldados en el propio devenir del pensamiento acerca de la realidad natural. De hecho, las nociones de prioridad y dependencia ontológica tienen una venerable historia en el desarrollo de la filosofía. En la relación platónica entre ideas y cosas sensibles, o en la distinción aristotélica entre sustancia primera y atributos, hay siempre ciertos ítems ontológicos - ideas, sustancias primeras - que tienen prioridad ontológica respecto de los restantes en el sentido de que no necesitan de otra cosa para existir; los demás ítems tienen una existencia secundaria, puesto que requieren los ítems primarios para su existencia - las cosas sensibles son meras "copias" de las ideas, los atributos se aplican a la sustancia primera. A comienzos de la modernidad, la dependencia ontológica se presenta como la relación entre cualidades primarias, dotadas de prioridad ontológica en tanto pertenecientes al objeto en sí mismo, y cualidades secundarias, resultantes del modo en que el objeto afecta nuestra sensibilidad. En todos estos casos, no se afirma que los ítems secundarios no existan o sean meramente ilusorios, sino que poseen una existencia derivada que depende de la existencia de los ítems primarios. La noción de dependencia ontológica puede expresarse en términos contrafácticos: que una ontología A dependa ontológicamente de una ontología B significa que si B no existiera, A tampoco existiría (sobre este punto, cf. la discusión de Needham, 2006 y la respuesta en Lombardi \& Labarca, 2006).

Por supuesto, no hay métodos para decidir el valor de verdad de un condicional contrafáctico más allá de toda duda. Sin embargo, lo que sucede en el plano de las teorías puede suministrarnos buenos argumentos para defender ciertas conclusiones en el plano ontológico. Esto es, podríamos reformular el contrafáctico en el plano ontológico, 'Si B no existiera, A tampoco existiría', en el plano epistemológico: 'Si la teoría B estuviese equivocada (fuese no exitosa, inadecuada), la teoría A también lo estaría'. En esta versión, el enunciado pierde gran parte de su carácter indecidible en la medida en que podemos consultar a la ciencia misma para estimar su valor de verdad. Es precisamente sobre la base de argumentos científicos que Jaap van Brakel consideraría tal enunciado como falso en muchos casos de interés:

Si la mecánica cuántica resultara equivocada, esto no afectaría todo (o incluso ningún) conocimiento químico acerca de las moléculas (unión, estructura, valencia etc.). Si la química molecular resultara equivocada, esto no descalificaría todo (o incluso ningún) conocimiento acerca, digamos, del agua (Brakel, 2000, p. 177). 
Cuando prestamos atención a la historia de la ciencia, la idea de van Brakel no queda exclusivamente confinada al ámbito de lo hipotético. Tomemos como ejemplo, precisamente, el desarrollo de la termodinámica, que nace como teoría científica con el trabajo de Carnot de 1824 y, a partir de ese momento, continúa desarrollándose en su descripción de los fenómenos térmicos. ${ }^{10}$ Dado el éxito hasta ese momento incuestionado de la mecánica clásica, en las últimas décadas del siglo xix se impone la idea de reducción en el nuevo campo de investigación: los fenómenos térmicos deberían poder ser explicados sobre la base del comportamiento mecánico de las partículas componentes del sistema bajo estudio. A la luz de esta idea, Boltzmann y Gibbs, entre otros, formularon la mecánica estadística clásica así como los nexos entre la nueva teoría y la termodinámica macroscópica. Tales nexos, si bien no de tipo reductivo en el sentido de Nagel (cf. Sklar, 1993), "funcionaron" bastante bien desde un punto de vista teórico y fueron incorporados al cuerpo de conocimientos de la física. En ese momento, quien creyera en nexos ontológicos de dependencia, habría hallado en las relaciones interteóricas recién formuladas el síntoma inequívoco de la dependencia ontológica de los fenómenos térmicos respecto de los hechos mecánicos subyacentes. Sin embargo, en las primeras décadas del siglo xx, la mecánica clásica comenzó a ser desplazada por la mecánica cuántica como teoría fundamental; como resultado de ello se desarrolló la mecánica estadística cuántica y, a partir de ese momento, los nexos interteóricos se establecieron con la nueva mecánica estadística, una vez más con resultados científicamente convincentes. ¿Qué queda, entonces, del supuesto acerca de la dependencia ontológica de los fenómenos térmicos respecto de la ontología clásica? Pero el aspecto central a destacar aquí es que la termodinámica, en tanto teoría científica que se ocupa de entidades y propiedades como calor, temperatura, entropía etc., no resultó afectada por todo ese proceso histórico, el reemplazo de la teoría "fundamental" no modificó los conceptos ni las leyes básicas de la termodinámica. Recordemos, además, que las primeras formulaciones teóricas en termodinámica, propuestas por Carnot, fueron desarrolladas adoptando la teoría del calórico como descripción de la ontología subyacente: nada cambiaría en los textos de termodinámica "fenomenológica", si postuláramos que el calor es un fluido elástico y sutil que llena todos los cuerpos, tal como afirmaba la teoría del calórico. Sobre esta base, no hay razón alguna para creer que un eventual cambio futuro en la teoría supuestamente fundamental afectará de manera significativa las descripciones termodinámicas. Si mañana se sustituyera la mecánica cuántica por una nueva teoría, esto no modificaría en absoluto nuestro conocimiento

10 No consideramos aquí las máquinas de vapor de Watt y Newcombe, construidas sin un marco teórico que las respaldara. 
sobre el calor, la temperatura o la entropía, ni nuestra manera de manipular y predecir fenómenos térmicos, en la medida en que tales conocimientos y prácticas responden a una teoría bien articulada y empíricamente exitosa como la termodinámica.

En definitiva, podríamos tener buenas razones para admitir la dependencia ontológica de los ítems "secundarios", si el abandono o la modificación de la teoría supuestamente fundamental implicara cambios profundos en la teoría que describe tales ítems: podría sostenerse, entonces, que las características de la ontología "secundaria" dependen o son, de algún modo, el resultado de las características de la ontología "fundamental". Pero si la suerte de la teoría "secundaria" o "fenomenológica" puede ser inmune a la suerte que corra la teoría "fundamental", no parece haber buenos motivos filosóficos para suponer que la ontología descrita por la primera depende ontológicamente de la ontología descrita por la segunda. Volviendo al caso de la termodinámica, si los términos termodinámicos no adquieren su significado a través de sus relaciones - reductivas o no - con los términos de la mecánica estadística - clásica o cuántica -, es razonable admitir que la existencia de los ítems termodinámicos no depende de la existencia de los ítems descritos por la mecánica estadística. En otras palabras, la temperatura de un gas no es energía cinética media de moléculas ni depende de tal energía cinética media. La temperatura es aquello que la termodinámica describe como medido por termómetros, que mantiene ciertas relaciones con la presión y el volumen, que se uniformiza siguiendo una ley definida etc. y estos hechos no requieren una ontología subyacente para seren reales.

En definitiva, sea sobre la base de supuestos metafísicamente reduccionistas o emergentistas, las interpretaciones tradicionales de la irreversibilidad siguen ancladas a la idea de relaciones asimétricas entre los diferentes dominios de lo real: existe un estrato "fundamental" - en nuestro caso, el dominio mecánico microscópico - dotado de prioridad ontológica respecto del estrato "fenomenológico" - en nuestro caso, el dominio termodinámico - que, o bien es una mera apariencia debido a nuestros limitados medios de acceso a lo real, desde la perspectiva reduccionista, o bien cuenta con una existencia derivada y dependiente del estrato fundamental, desde la perspectiva emergentista. Sin embargo, no hemos hallado motivos convincentes, ni formales ni histórico-filosóficos, que respalden este supuesto de asimetría. Si pretendemos, entonces, prescindir de tal supuesto metafísico, debemos contar con un marco filosófico que nos permita admitir la coexistencia de diferentes ontologías, sin prioridades ni dependencias; este marco es el que nos brinda un pluralismo ontológico de raigambre kantiana. 


\section{Pluralismo ontológigo de Raíces Kantianas}

Antes de Kant, la polémica giraba en torno de si hay realmente objetos materiales independientes de la mente humana, o si sólo existe lo mental (por ejemplo, Locke versus Berkeley). Kant comienza por rechazar los supuestos de esta discusión entre "realistas trascendentales" e "idealistas empíricos", como él los llamaba, y plantea el problema del realismo en otros términos. No se trata de establecer qué es lo que realmente existe en sí mismo, sino de aceptar que todo aquello que llamamos "objeto" está constituido en el marco de nuestro esquema categorial. Por lo tanto, si bien se admite la existencia de una realidad independiente del sujeto, no podemos formar ninguna idea o concepto de los componentes de dicha realidad, es decir, de las "cosas-en-sí". "Como afirma Torretti no sólo no hay una correspondencia biunívoca entre cosas-en-sí y objetos de la experiencia, sino que tampoco puede suponerse que cada una de las determinaciones de los objetos empíricos encuentra su razón de ser en la cosa-en-sí. Quien asumiera este supuesto tendría que admitir que "Kant ha derogado de una plumada toda su enseñanza acerca de la limitación de nuestro conocimiento" (Torretti, 2005a, p. 664) al aceptar que "el conocimiento de aquéllas [las determinaciones del fenómeno] constituirá un saber satisfactorio sobre ésta [la cosa en sí], al menos tan adecuado como la ciencia a que aspira el realista precrítico que toma los fenómenos por cosas en sî" (2005a, p. 665).

En su libro, Razón verdad e historia, Hilary Putnam adopta explícitamente una posición de corte kantiano a la que denomina "internalismo", que se opone al "externalismo" o "realismo metafísico" al cual suele referirse como "la perspectiva del ojo de Dios". Según el realismo metafísico, la realidad es una totalidad fija de objetos que existen independientemente de nuestro conocimiento. Por lo tanto, hay una única descripción verdadera y completa del mundo, cuya verdad consiste en la correspondencia entre las palabras y los objetos independientes del sujeto. Para determinar dicha verdad, el realismo metafísico requiere un punto de vista no humano, el ojo de Dios, desde el cual acceder a la realidad en sí misma.

El internalismo, por el contrario, adopta la noción kantiana de objeto empírico, según la cual los objetos no poseen existencia independiente del sujeto cognoscente: "Los 'objetos' no existen independientemente de los esquemas conceptuales. Desmenuzamos el mundo en objetos cuando introducimos uno u otro esquema descriptivo" (Putnam, 1988 [1981] , p. 52). A esto se refiere Putnam cuando afirma que la pregunta “¿de qué objetos consta el mundo?" sólo cobra sentido si se formula desde dentro de un esquema conceptual. Es siempre a través de un cierto sistema de conceptos que nos enfrentamos al mundo y lo categorizamos: de tal síntesis surgen los objetos en tanto 
objetos, es decir, con una identidad. Como afirma Ransanz (1999, p. 209), los objetos dependen en un sentido fuerte, que incluye existencia, de los esquemas conceptuales, los cuales no son meros intermediarios entre sujetos y objetos sino que cumplen un papel central en la constitución de los objetos.

Ahora bien, aun cuando Kant

compara atinadamente al científico con un juez instructor que dirige sus preguntas a la naturaleza y fija los términos en que tiene que venir concebida la respuesta, no se pone en el caso de que, frustrado porque las respuestas se contradicen, las indagaciones se complican y atascan y, en general, no parecen estar llegando a nada, el juez instructor repiense sus preguntas, modifique las categorías en que deben encuadrarse las respuestas e incluso cambie las metas de la investigación o los criterios para evaluar sus resultados (Torretti, 2005b, p. 20).

Por ello, a la noción kantiana de objeto, el Putnam historicista le agrega la tesis de la relatividad conceptual, según la cual ningún concepto, ni siquiera las categorías más básicas, tiene una validez única o vigencia absoluta. No hay una ontología metafísicamente privilegiada, pues coexisten esquemas conceptuales alternativos, no convergentes ni reducibles a un esquema único. El fenómeno de la relatividad conceptual "depende del hecho de que los mismos primitivos lógicos y, en particular, las nociones de objeto y existencia, tienen una multitud de usos diferentes, y no un 'significado' absoluto" (Putnam, 1994 [1987], p. 19).

La tesis de la relatividad conceptual trae consigo un pluralismo ontológico, pues abre la posibilidad de tener concepciones del mundo con ontologías distintas, incluso incompatibles, que resulten igualmente adecuadas en ciertos contextos. El pluralismo ontológico ha sido esgrimido contra el realismo metafísico de corte cientificista, según el cual la ciencia converge hacia la verdadera descripción de lo real. Pero este enfoque filosófico también puede ser aplicado en un sentido sincrónico: aún en el contexto de un mismo paradigma pueden coexistir diversas ontologías que, si bien pueden encontrarse interrelacionadas, incluyen ítems diferentes, no reductibles entre sí y sin prioridades o dependencias ontológicas (para un desarrollo pormenorizado del realismo pluralista de raíces kantianas, cf. Lombardi \& Ransanz, en prensa).

Esta posición filosófica resulta particularmente fructífera cuando el problema consiste en interpretar las relaciones entre ontologías descriptas por teorías científicas igualmente exitosas desde un punto de vista empírico. En física, por ejemplo, suele darse la situación de dos teorías que brindan descripciones incompatibles de un mismo sistema, puesto que una le adjudica una propiedad $P$ y la otra le adjudica su nega- 
ción $\neg P .{ }^{11}$ Por ejemplo, éste es el caso de los sistemas dinámicos altamente inestables, que resultan deterministas en su descripción microscópica pero indeterministas en su descripción macroscópica. La situación reaparece en el límite clásico de la mecánica cuántica, donde el problema consiste en compatibilizar la existencia de los objetos separables de las teorías clásicas con la ontología no separable de la mecánica cuántica. Esto es precisamente lo que sucede en nuestro problema de la irreversibilidad. El carácter irreversible y no unitario del comportamiento temporal del sistema en el nivel macroscópico debe compatibilizarse con una dinámica subyacente reversible y unitaria. En todos estos casos, el externalista que no rehuye a la discusión ontológica está comprometido a decidir cuál de las dos propiedades es real o, al menos, fundamental: ¿es el sistema determinista o indeterminista? ¿Reversible o irreversible? ¿Sustancial o contextual?

Como hemos señalado, las respuestas tradicionales a estas preguntas se basan en suponer el carácter "fundamental" de la ontología microscópica, de este modo, el indeterminismo y la irreversibilidad de los procesos macroscópicos y la naturaleza de los objetos clásicos se convierten en meras apariencias subjetivas o, al menos, en fenómenos dependientes de una realidad subyacente que posee las características precisamente opuestas. Estas posiciones, que postulan una relación asimétrica entre ontologías, adoptan implícitamente una posición metafísicamente realista, según la cual el mundo, con sus categorías ontológicas básicas, los ítems que lo habitan y, eventualmente, los niveles en los que se organiza, existe con independencia del sujeto cognoscente: la realidad - fundamental, en el caso reduccionista, o desplegada en diferentes niveles jerarquizados, en el caso emergentista - está “allí afuera”, a la espera de ser conocida. Sin embargo, también hemos argumentado que el supuesto de la prioridad ontológica de un estrato de lo real respecto de los restantes estratos, sean éstos aparentes o derivados, carece de motivaciones filosóficas convincentes. Frente a ello, el realismo pluralista de filiación kantiana prescinde de todo supuesto metafísico de prioridad y dependencia, para admitir la coexistencia de múltiples ontologías autónomas. La existencia objetiva de cada una de ellas no depende de sus relaciones con otras ontologías, sino de la teoría cuyo esquema conceptual interviene en su constitución.

Este pluralismo ontológico ha sido aplicado de un modo fecundo a diferentes problemas específicos de la filosofía de la física. Por ejemplo, ha sido utilizado para

11 El hecho de que dos teorías refieran a ontologías incompatibles no implica que ambas no puedan identificar el mismo sistema. En efecto, los esquemas conceptuales correspondientes a tales teorías pueden compartir ciertas categorías o conceptos muy básicos (por ejemplo, espacio y tiempo) que permitan identificar un sistema desde ambas teorías como el mismo (por ejemplo, a través de su localización espacio-temporal), a pesar de que los restantes conceptos conduzcan a ontologías incompatibles (para una discusión detallada sobre este punto, cf. Lombardi \& Ransanz, en presa). 
enfrentar el problema de la compatibilidad entre descripciones deterministas e indeterministas en sistemas altamente inestables (cf. Lombardi, 2002a; 2002b), para explicar la irreversibilidad cuántica a partir de una dinámica regida por la ecuación de Schrödinger y, por tanto, reversible y unitaria (cf. Castagnino, Gadella \& Lombardi, 2005, 2006), y para dar cuenta del límite clásico de la mecánica cuántica, esto es, del modo en que puede obtenerse una descripción clásica a partir de la descripción cuántica de un sistema físico (cf. Castagnino \& Lombardi, 2003, 2005b). En filosofía de la química, ha sido útil para abordar el problema de las relaciones entre el mundo físico y el mundo químico, tradicionalmente interpretadas en términos de reducción (cf. Lombardi \& Labarca, 2005b, 2006), y también para elucidar el estatus del concepto de orbital atómico (cf. Lombardi \& Labarca, 2007). Desde una perspectiva ontológicamente pluralista, el indeterminismo, la irreversibilidad cuántica, el mundo clásico y los fenómenos químicos son tan reales y objetivos como las entidades, propiedades y procesos del dominio microscópico. Y lo son en función de los esquemas conceptuales que los constituyen como objetos de la experiencia, con independencia de la suerte que corran las teorías que describen la ontología microscópica subyacente.

Sobre la base de lo discutido en los apartados anteriores, el problema de la irreversibilidad en mecánica estadística clásica se suma a los casos de aplicación fecunda del pluralismo ontológico. Desde esta perspectiva, la existencia objetiva de la irreversibilidad macroscópica no depende en modo alguno de su vinculación con procesos microscópicos: se trata de una propiedad real y autónoma, perteneciente a la ontología constituida por el esquema conceptual de una teoría exitosa como la termodinámica, y que no necesita referirse a un nivel subyacente para adquirir legitimidad ontológica. Aun si careciéramos de la mecánica clásica para referirnos al nivel microscópico, incluso si dicho nivel no existiera y los fenómenos térmicos se explicaran en términos de una sustancia como el calórico, la termodinámica continuaría describiendo de un modo empíricamente adecuado la irreversibilidad de procesos como, por ejemplo, la difusión de materia o la uniformización de temperaturas. Por otra parte, tampoco hay motivo alguno para poner en duda la objetividad de la descripción mecánica microscópica y, por tanto, el carácter real de la reversibilidad de los procesos mecánicos. Pero, una vez que hemos renunciado a la perspectiva del ojo de Dios, ya no estamos forzados a privar de objetividad o a dotar de un estatus ontológico secundario a todas salvo una de las descripciones de un sistema físico. El hecho de que exista, como nos muestra el enfoque de Gibbs, un nexo específico entre evoluciones reversibles e irreversibles no conduce a postular una relación asimétrica entre ambas. Por el contrario, tal nexo puede concebirse como un vínculo simétrico entre diferentes ontologías de existencia autónoma. De este modo, nuestro mundo científico se nos presenta como una realidad diversificada, que se despliega en múltiples ontologías autónomas pero no por ello inconexas. 


\section{Conclusiones}

Transcurrido más de un siglo desde su aparición en la escena científica y filosófica, el problema de la irreversibilidad continúa siendo uno de los grandes desafíos de la filosofía de la física. A lo largo de los años, muchos físicos y filósofos se han ocupado del problema; sin embargo, en general, las discusiones se han visto opacadas por la inadecuada elucidación de los conceptos básicos involucrados en el problema y por supuestos metafísicos no debidamente explicitados.

En el presente trabajo hemos intentado, en primer lugar, poner un poco de orden en la discusión, brindando una caracterización precisa del problema de la irreversibilidad. Tal caracterización muestra que las dificultades para dar cuenta de la irreversibilidad termodinámica macroscópica derivan, no de la t-invariancia de las leyes microscópicas, como suele afirmarse, sino de la unitariedad de las evoluciones mecánicas. Sobre esta base, hemos formulado el problema en el ámbito de la mecánica estadística clásica, donde la irreversibilidad macroscópica se vincula con la microdinámica reversible y unitaria a través de una relación de grano grueso, relación tradicionalmente interpretada como expresión formal del carácter subjetivo u ontológicamente derivado de los fenómenos irreversibles. Frente a estas interpretaciones, hemos argumentado que nada hay en la relación misma que fuerce la prioridad ontológica del mundo de la mecánica clásica respecto del dominio termodinámico: nada impone una relación asimétrica entre las respectivas ontologías. Por lo tanto, la arraigada imagen ontológica que presenta al mundo clásico como "fundamental" y al dominio termodinámico como meramente "secundario" y "fenomenológico" no es más que el resultado de un supuesto metafísico que carece de fundamentos formales e histórico-filosóficos. Finalmente, hemos argumentado que es posible prescindir de tal supuesto desde un pluralismo ontológico basado en un realismo de raigambre kantiana, que nos permite admitir la coexistencia de diferentes ontologías, sin la postulación de identificaciones reductivas, prioridades o privilegios. Desde esta perspectiva, la irreversibilidad termodinámica macroscópica resulta ser una propiedad tan real como la reversibilidad mecánica. Los procesos irreversibles que observamos a diario dejan de ser meras apariencias ilusorias o fenómenos secundarios dependientes de una ontología última y fundamental. $\$$ 
Agradecimientos. Este trabajo fue posible gracias al apoyo brindado por el Consejo Nacional de Investigaciones Científicas y Técnicas (CONICET), la Agencia Nacional de Promoción Científica y Tecnológica, la Universidad del Centro Educativo Latinoamericano y la Universidad Nacional de Quilmes, Argentina.

\title{
Martín LABARGA
}

Professor de la Universidad Nacional de Tres de Febrero, Universidad Nacional de Quilmes, Argentina. mglabarca@unq.edu.ar

\section{Olimpia Lombardi}

Investigadora de CONICET, Universidad de Buenos Aires, Argentina.

olimpiafilo@arnet.com.ar

\begin{abstract}
The problem of irreversibility finds its roots at the end of 19th century and the beginning of 2oth century with the works of Maxwell, Boltzmann and Gibbs. The controversy arises when trying to make compatible the irreversible macroscopic evolution of thermodynamics systems with $\mathrm{t}$-invariant dynamics of its microscopic components. In the traditional approach of statistical mechanics, the irreversible evolution is explained by introducing a coarse grain on the underlying microscopic dynamics. For this reason, it is generally accepted that statistical mechanics offers a subjective description of irreversibility. The purpose of this work is to reject the traditional approaches of the problem by stressing that its only foundation is the implicit adoption of metaphysical realism that adjudges ontological priority to the microscopic world. We will argue that the problem can be addressed on the basis of an ontological pluralism of kantian roots, inspired by Putnam's internalist realism. From this philosophical framework, macroscopic irreversibility is an objective property that has not need to be explained in microscopic terms to acquire ontological legitimacy: the coarse grain is the relationship between two equally objective descriptions.
\end{abstract}

KEYWORDS • Irreversibility. Statistical mechanics. Gibbs. Ontological pluralism. Coarse grain.

\section{REFERENGIAS BIBLIOGRÁFICAS}

Albert, D. Time and chance. Cambridge: Harvard University Press, 2000.

Arntzenius, F. Time reversal operations- representations of the Lorentz group, and the direction of time. Studies in History and Philosophy of Modern Physics, 35, p. 31-44, 2004.

Atmanspacher, H. \& Bishop, R. (Ed.). Between chance and choice: interdisciplinary perspectives on determinism. Thorverton: Imprint-Academic, 2002.

Stability conditions in contextual emergence. Chaos and Complexity Letters, 2, p. 139-50, 2007.

Bhushan, N. \& Rosenfeld, S. (Ed). Of minds and molecules. New philosophical perspectives on chemistry. New York: Oxford University Press, 2000. 
Born, M. Natural philosophy of cause and chance, Oxford: Clarendon Press, 1949.

Brakel, J. van. The nature of chemical substances. In: Bhushan, N. \& Rosenfeld, S. (Ed). Ofminds and molecules. New philosophical perspectives on chemistry. New York: Oxford University Press, 2000. p. $162-84$.

Bricmont, J. Science of chaos or chaos in science? Physicalia Magazine, 17, p. 159-208, 1995.

BroAd, C. D. The mind and its place in nature. New York: Kegan Paul, 1925.

Bunge, M. Is chemistry a branch of physics? Zeitschrift fur allgemeine Wissenschaftstheorie, 13, p. 209-23, 1982.

Castagnino, M.; Gadelda, M. \& Lombardi, O. Time's arrow and irreversibility in time-asymmetric quantum mechanics. International Studies in the Philosophy of Science, 19, p. 223-43, 2005.

. Time-reversal, irreversibility and arrow of time in quantum mechanics. Foundations of Physics, 36, p. 4.07-26, 2006.

Castagnino, M.; Lara, L. \& Lombardi, O. The cosmological origin of time-asymmetry. Classical and Quantum Gravity, 20, p. 369-91, 2003.

Castagnino, M. \& Lombardi, O. Self-induced decoherence- a new approach. Studies in History and Philosophy of Modern Physics, 35, p. $7^{3-107,} 2003$.

. A global and non entropic approach to the problem of the arrow of time. In: Reimer, A. (Ed.). Spacetime physics research trends. Horizons in world physics. New York: Nova Science, 2005a. p. 74-108.

. Self-induced decoherence and the classical limit of quantum mechanics. Philosophy of Science, $72, p \cdot 7^{6} 4^{-} 7^{6}, 2005^{b}$.

Courbage, M. \& Prigogine, I. Intrinsic randomness and intrinsic irreversibility in classical dynamical systems. Proceedings of the NationalAcademy of Sciencies of the United States of America, 8o, p. 24,12-6, 1983.

EARMAn, J. What time reversal invariance is and why it matters. International Studies in the Philosophy of Science, 16, p. 245-64, 2002.

EL HANI, C. N. Os debates sobre a emergéncia de propriedades: onde estamos atualmente? In: Rabossi, E. (Ed.). La mentey sus problemas. Temas actuales de filosofía de la psicología. Buenos Aires: Catálogos, 2004. p. 24.9-300.

HeISENBERG, W. The physicist's conception of nature. London: Hutchinson, $195^{8}$.

HENDRY, R. Molecular models and the question of physicalism. Hyle. International Journal for Philosophy of Chemistry, 5, p. 14,3-60, 1999.

Koopman, B. O. Hamiltonian systems and transformations in Hilbert space. Proceedings of the National Academy of Sciences, 17, p. 315-8, 1931 .

Lombardi, O. Prigogine: ciencia y realidad. Crítica. Revista Hispanoamericana de Filosofía, 3o, p. 47-75, 1998.

. El fin de la ommnisciencia: la respuesta de Prigogine al problema de la irreversibilidad. Theoria. Revista de Teoría, Historia y Fundamentos de la Ciencia, 14, p. 489-510, 1999.

. La interpretación de la irreversibilidad: Prigogine versus Gibas. Diálogos, 35, p. 37-56, 2000.

. Determinism, internalism and objectivity. In: Atmanspacher, H. \& Bishop, R. (Ed.). Between chance and choice: interdisciplinary perspectives on determinism. Thorverton: Imprint-Academic, $2002 \mathrm{a}$. p. $75^{-8} 7$.

. Caos, ergodicidad e internalismo. Revista Latinoamericana de Filosofía, 28, p. 7-33, 2002b.

. Irreversibilidad: origen y vigencia de una controversia científica. Revista Patagónica de Filosofía, 3 , p. 23-4, 2003a.

. El problema de la ergodicidad en mecánica estadística. Crítica. Revista Hispanoamericana de Filosofía,

35 , p. 3-4, 2003 b. 
LOMBARdi, O. T-invariancia, irreversibilidad, flecha del tiempo: similares pero diferentes. In: MARTins, R. A.; Boido, G. \& Rodríguez, V. (Ed.). Fisica: estudios filosóficos e históricos. Campinas: Asociación de Filosofía e Historia de la Ciencia del Cono Sur (AFHIC), 2006. p. 85-117.

Lombardi, O. \& LABARCA, M. Los enfoques de Boltzmann y de Gibbs frente al problema de la irreversibilidad. Crítica. Revista Hispanoamericana de Filosofía, 37, p. 39-81, 2005a. . The ontological autonomy of the chemical world. Foundations of Chemistry, 7, p. 125-4, $2005 \mathrm{~b}$.

. The ontological autonomy of the chemical world: a response to Needham. Foundations of Chemistry, 8, p. 81-92, 2006.

. The philosophy of chemistry as a new resource for chemistry education. Journal of Chemical Education, 84, p. $187^{-92,2007 .}$

Lombardi. O. \& Ransanz, A. R. P. Los múltiples mundos de la ciencia. En prensa.

Luisi, P. L. Emergence in chemistry: chemistry as the embodiment of emergence. Foundations ofChemistry, 4, p. 183-200, 2002.

MackeY, M. C. The dynamic origin of increasing entropy. Review of Modern Physics, 61, p. 981-1015, 1989.

Martins, R. A.; Boido, G. \& Rodríguez, V. (Ed.). Física: estudios filosóficos e históricos. Campinas: Asociación de Filosofía e Historia de la Ciencia del Cono Sur (AFHIC), 2006.

Misra, B.; Prigogine, I. \& Courbage, M. From deterministic dynamics to probabilistic descriptions. Physica A, 98, p. 1-26, 1979.

Needham, P. Ontological reduction: comment on Lombardi-Labarca. Foundations of Chemistry, 8 , p. $73-80,2006$.

Popper, K. Teoría cuántica y el cisma en física. Madrid: Tecnos, 1982 [1956].

Prigogine, I. Les lois du chaos. Paris: Flammarion, 1994.

Prigogine, I. \& Stengers, I. La nueva alianza, metamorfosis de la ciencia. Madrid: Alianza Editorial, 1983 [1979].

Putnam, H. Razón, verdad e historia. Madrid: Tecnos, 1988 [1981]. Las mil caras del realismo. Barcelona: Paidós, 1994 [1987].

Rabossi, E. (Ed.). La mente y sus problemas. Temas actuales de filosofía de la psicología. Buenos Aires: Catálogos, 2004.

Ransanz, A. R. Kuhny el cambio científico: México: Fondo de Cultura Económica, 1999.

Reimer, A. (Ed.). Spacetime physics research trends. Horizons in world Physics. New York: Nova Science, 2005 .

Rон RLICH, F. Pluralistic ontology and theory reduction in the physical sciences. British Journal for the Philosophy of Science, 39, p. 295-312, 1988.

SkLaR, L. Physics and chance, Cambridge: Cambridge University Press, 1993.

Torretri, R. Manuel Kant. Santiago de Chile: Ediciones Universidad Diego Portales, 2005a. . Conocimiento discursivo. Lección Inaugural dictada en la Universidad Autónoma de Barcelona el 28 de Abril de 2005, en ocasión de la investidura del autor como Doctor Honoris Causa de dicha Universidad, 2005b. Reeditado en Revista UDP, 2, p. 67-72, 2005.

VAUCLAIR, S. Eléments de physique statistique. Paris: Interéditions, 1993. 